\title{
Review Article \\ Control Application of Piezoelectric Materials to Aeroelastic Self-Excited Vibrations
}

\author{
Mohammad Amin Rashidifar and Darvish Ahmadi \\ Department of Mechanical Engineering, Islamic Azad University, Shadegan Branch, Fatholmobin Bl, \\ Shadegan, Khuzestan 6431863758, Iran \\ Correspondence should be addressed to Mohammad Amin Rashidifar; rashidifar_58@yahoo.com
}

Received 2 November 2013; Revised 14 February 2014; Accepted 2 March 2014; Published 17 March 2014

Academic Editor: Hongyi Li

Copyright (C) 2014 M. A. Rashidifar and D. Ahmadi. This is an open access article distributed under the Creative Commons Attribution License, which permits unrestricted use, distribution, and reproduction in any medium, provided the original work is properly cited.

\begin{abstract}
A method for application of piezoelectric materials to aeroelasticity of turbomachinery blades is presented. The governing differential equations of an overhung beam are established. The induced voltage in attached piezoelectric sensors due to the strain of the beam is calculated. In aeroelastic self-excited vibrations, the aerodynamic generalized force of a specified mode can be described as a linear function of the generalized coordinate and its derivatives. This simplifies the closed loop system designed for vibration control of the corresponding structure. On the other hand, there is an industrial interest in measurement of displacement, velocity, acceleration, or a contribution of them for machinery condition monitoring. Considering this criterion in quadratic optimal control systems, a special style of performance index is configured. Utilizing the current relations in an aeroelastic case with proper attachment of piezoelectric elements can provide higher margin of instability and lead to lower vibration magnitude.
\end{abstract}

\section{Introduction}

The dependence of mechanical and electrical properties of piezoelectric materials on their application in various materials as patches or layers makes them an appropriate sensor or actuator for vibration control of structures. In sensing situation, the mechanical and creep deformations of structures can be determined by measuring the electrical potential produced in piezoelectric materials. This property is termed the direct property of the piezoelectric material. Then an effective feedback mechanism sends an electric signal to an actuator to keep the vibration of the mechanical system to a minimum. In actuator applications, the inverse piezoelectric effect is used. Recently, this technique is widely used in the active control of vibrations, deformation control of structures, and aerospace industries.

This technique has been investigated by research in solid and aeroelastic areas. In solid, Gaudenzi et al. [1] investigated the vibration control of an overhung beam by means of finite element approach based on Euler-Bernoulli beams. They studied state feedback and velocity feedback control of vibration. Moreover, Q. Wang and C. M. Wang [2] implemented vibration control of a beam with piezoelectric patches by taking finite element method into account. They determined an optimized position for an appropriate actuator and the subsequent vibration amplitude of a hinged-hinged beam by applying a feedback control procedure and converting the finite element model into its state space form. Narayanan and Balamurugan [3] studied the effects of temperature on mechanical and electrical properties of beams in addition to vibration control of them. They applied LQR differentiator controller and optimal control for this purpose. In another research, piezoelectric materials were used by $\mathrm{Xu}$ and Koko [4] as an actuator for vibration control of a beam, and the optimum position of the actuators was determined by introducing a controllability index. Lin and Nien [5] used an analytical method for active vibration control of an overhung beam. In the latest reference, six pairs of piezoelectric patches were used as sensor and actuator.

There are some historical reports about the aeroelastic application of the beam model. Aeroelasticity is an interaction between elastic deformation of structures in an air stream and the resulting aerodynamic forces. The widespread application of piezoelectric materials in various areas such as shape control, sound and vibration damping, and fault diagnosis of structures facilitates the use of these materials 
in controlling the vibrations due to wings flutter. JacquetRichardet [6] represented some relations for analyzing the aeroelastic behavior of beam models of blades. Karadal et al. [7] introduced a state space formula for solution of aeroelastic problems based on rational approximation technique which can facilitate aeroservoelasticity (aeroelasticity control) of wings. Concerning the differences between the wings and gas turbine blades, the bladed disk systems of gas turbines differ from the wings systems in internal flow between the blade paths and in producing traveling waves due to the cyclic nature of bladed disk systems. Considering these differences, Rahi et al. [8] applied timoshenko beam theory and the rational approximation technique for flutter analysis of gas turbine blades [8]. Flutter is an unstable and self-excited vibration of a body in an air stream and results from a continuous interaction between aerodynamics and structural mechanics.

Regarding the above-mentioned histories, it seems that there is a need for proposing a method for vibration control of blades by piezoelectric materials is introduced. This study aims to introduce such a method. To this end an overhung beam (blade) is equipped with piezoelectric patches. Controllability of the beam equipped with piezoelectric elements is evaluated initially, and the active vibration control of such beam is discussed by velocity feedback and optimal control system under an initial linear LQR displacement. Several parameters such as the optimized position of the sensor and $[Q] \&[R]$ actuators and the effects of weighting matrices are investigated and calculated. The governing differential equation of motion is then rewritten in state space form. Following this procedure, the proper size and position of the piezoelectric elements can be determined for a certain configuration of blades.

\section{Theory of Piezoelectricity}

In sensor situation, when a stress is applied to a crystal, it causes a polarization which can be described by

$$
\begin{gathered}
\{S\}=[s]\{T\}+[g]\{D\}, \\
\{E\}=[g]\{T\}+[\beta]^{T}\{D\} .
\end{gathered}
$$

Similarly, an electric field applied to the crystal will make it become strained. This should reduce the vibration of mechanical systems. The following relations can describe the behavior of such systems:

$$
\begin{gathered}
\{S\}=[s]\{T\}+[d]\{D\}, \\
\{E\}=[d]\{T\}+[\varepsilon]^{T}\{D\},
\end{gathered}
$$

where the nomenclature used is as follows: $S$ : strain; $T$ : stress; $D$ : electric displacement; $d$ : piezoelectric constant; $E$ : electric field; $g$ : piezoelectric constant; $\varepsilon$ : dielectric constant; $C$ : stiffness matrix; $d=C^{-1} e$ and $s=C^{-1}$.

\section{System Model}

An overhung beam with piezoelectric patches as sensor and actuator is considered for analysis, as shown in Figure 1. Piezoelectric layers can also be used in the model.

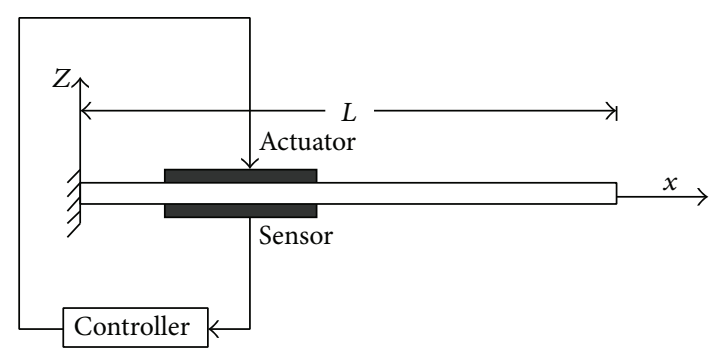

FIGURE 1: An overhung beam with typical piezoelectric patches.

By the application of assumed mode approach and the normalized mode with respect to the mass and to the vibration response, the following equation will be obtained:

$$
\left[M_{s}\right] \ddot{q}+\left[C_{s}\right] \dot{q}+\left[K_{s}\right] q=\left[F_{d}\right]+\left[P_{a G}\right]\left\{\phi_{a}(t)\right\},
$$

in which $\left[M_{s}\right],\left[C_{s}\right]$, and $\left[K_{s}\right]$ are generalized mass, damping, and stiffness matrix, respectively. $F_{d}$ is a generalized external force and $\phi_{a}$ is an induced voltage. The above relation is written in matrix form because pitching stress is also added. The value of $P_{a}$ is obtained by the following relation:

$$
P_{a}=E_{a} \cdot d_{31 a} \cdot b \cdot r_{a} .
$$

$E_{a}$ is the modulus of elasticity of the actuator, $d_{31 a}$ is the dielectric actuator constant, $b$ is the beam width, and $r_{a}$ is the distance between the actuator axis and the neutral axis. $[M],[C]$, and $[K]$ are diagonal and square matrices due to orthogonality. $\left\{\phi_{a}\right\}$ is determined with substitution of the new aeroelastic parameters. Consider

$$
\ddot{\eta}+2 \zeta_{c} \omega_{c} \dot{\eta}+\omega_{c}^{2} \eta=\mu_{x} \omega_{c}^{2} q+\mu_{v} \omega_{c}^{2} \dot{q} .
$$

The state space form of this equation is preferred with the objective of having more suitable optimal control. So, the equations of the closed loop system can be rearranged to yield that

$$
\{\dot{\xi}\}=[A]\{\xi\}+[B]\left\{\phi_{a}\right\}+[\widehat{B}]\left\{u_{d}\right\},
$$

where $u_{d}$ is the inlet disturbance of the system and $\phi_{a}$ is the input control. $[B]$ And $\lfloor\widehat{B}\rfloor$ are matrices involving $P_{a}$ and $F_{d}$, respectively, the derivation of which will be described later. $\xi$ Is also defined as $\xi=\left\{\begin{array}{c}q \\ \dot{q}\end{array}\right\}$ in which $q=\left[\begin{array}{l}q_{h} \\ q_{\theta}\end{array}\right]$.

The output of the system can be demonstrated in the state space form as

$$
[y]=\left\lfloor C_{y}\right\rfloor\{\xi\} .
$$

\section{Control System Design}

4.1. Velocity Feedback Control. In this control method, the differential output signal of the sensor is calculated and is sent to the actuator in order to achieve the rate of the strain variations. The induced voltage of the actuator can be determined by

$$
\phi_{a}(t)=-G_{c} \dot{\phi}_{s}(t) .
$$

As the feedback control can increase the damping of such systems, the vibration amplitude can be properly reduced by this approach. $G_{c}$ is a definite number determined in design. 
4.2. Linear Optimal Control. Linear optimal control is used for determination of the most suitable control coefficients, and the feedback control system is designed for minimizing the quadratic energy function and the quadratic error function. A performance index is defined as

$$
J=\int_{0}^{\infty}\left(\{X\}^{T}[Q]\{x\}+\left\{\phi_{a}\right\}^{T}[R]\left\{\phi_{a}\right\}\right) d t
$$

in which $[Q]$ and $[R]$ are weighting positive and negative semidefinite matrices. Hence, assuming a complete state controllability, the induced voltage can be obtained by the following relation:

$$
\left\{\phi_{a}\right\}=-\left[G_{c}\right]\{\xi\}
$$

where

$$
G_{c}=-[R]^{-1}[B]^{T}[\widehat{P}] .
$$

$[B]$ can be achieved by

$$
B=\left\{\begin{array}{c}
0 \\
{\left[P_{a}\right]}
\end{array}\right\} .
$$

The optimized $[\widehat{P}]$ must satisfy the Riccati equation which is

$$
\begin{aligned}
& {[A]^{T}[\widehat{P}]+[\widehat{P}][A]-[\widehat{P}][B][R]^{-1}[B]^{T}[\widehat{P}]} \\
& +\left[C_{0}\right]^{T}[Q]\left[C_{0}\right]=0 .
\end{aligned}
$$

After rearranging, the governing equation of the closed loop system becomes

$$
[\dot{\xi}]=\left([A]-[B]\left[G_{c}\right]\right)\{\xi\}+\lfloor\widehat{B}\rfloor\left\{u_{d}\right\}=\left[A_{c l}\right]+\lfloor\widehat{B}\rfloor\left\{u_{d}\right\},
$$

where $\left[A_{c l}\right]$ is a closed loop system matrix.

In flutter analysis, the main objective is to suppress flutter occurrence and consequently to shift the flutter speed far from the operating condition. This task can be done by attaching a proper amount of piezoelectric materials in the most suitable position. Such control systems can be used, for example, for one turbine blade row which has excessive vibration. However in turbomachinery, especially in gas turbines, the large number of structures can limit the amount of piezoelectric materials and energy considered for each of them. So the goal of the control system design is to develop a mechanism which would be relatively inexpensive, light weight, and capable of vibration reduction at a predominant frequency or in a frequency range to a certain amplitude. Hence, there must be a compromise between the actuator magnitude and the output of the system $\{y\}$ which is

$$
\{y\}=\left\lfloor C_{y}\right\rfloor\{\xi\}
$$

So,

$$
\left\lfloor C_{y}\right\rfloor=\left[\begin{array}{cc}
{[I]} & 0 \\
0 & {[I]}
\end{array}\right]
$$

On the other hand, in condition monitoring of machinery equipment, the main objective is to minimize the displacement, velocity, acceleration, or a combination of them, based on the frequency domain in which the predominant vibration occurred [9]. At low frequencies, the displacement amplitude is of interest because it is difficult to detect velocity and acceleration in these frequency ranges. However, at higher frequencies, velocity or in some particular circumstances acceleration is more effective because of their dependence on frequency. High frequency acceleration usually occurs in hypersonic condition which is not practical in this field. Sometimes $g$-peak acceleration is particularly used in the other dynamical systems such as antifriction bearing analysis. Therefore, the main output of the aeroelastic control system is displacement and velocity. Peak to peak, average, and RMS measurement may be used for evaluation based on the analysis purpose, but RMS is the most suitable, and it is widely used in many industries. So, a linear square contribution of displacement and velocity is developed. The coefficients are the corresponding amplifier gain of the parameters denoted by $\mu_{x}$ and $\mu_{v}$, respectively. Vibration analyzers can measure an infinite number of time waves in a finite time interval. Hence, for a certain mode, the performance index can be considered as a function of $e$ and $\dot{e}$ or can be written as

$$
\begin{aligned}
J= & \int_{0}^{T}\left(q^{T} \mu_{x 1} q+\dot{q}^{T} \mu_{v 1} \dot{q}\right) d t+\int_{T}^{2 T}\left(q^{T} \mu_{x 2} q^{T}+\dot{q}^{T} \mu_{v 2} \dot{q}\right) d t \\
& +\cdots+\int_{0}^{\infty}\left\{\phi_{a}\right\}^{T} R\left\{\phi_{a}\right\} d t, \\
& \mu_{x n}=\left\{\begin{array}{cc}
\mu_{x h n} & 0 \\
0 & \mu_{x \theta n}
\end{array}\right\}, \quad \mu_{v n}=\left\{\begin{array}{cc}
\mu_{v h n} & 0 \\
0 & \mu_{v \theta n}
\end{array}\right\} .
\end{aligned}
$$

The above relation can be summarized to a special quadratic performance index:

$$
\int_{0}^{\infty}\left(\{\xi\}^{T}[Q]\{\xi\}+\left\{\phi_{a}\right\}^{T}[R]\left\{\phi_{a}\right\}\right) d t,
$$

where for each mode

$$
[Q]=\left[\begin{array}{cccc}
\mu_{x h n} & 0 & 0 & 0 \\
0 & \mu_{x \theta n} & 0 & 0 \\
0 & 0 & \mu_{v h n} & 0 \\
0 & 0 & 0 & \mu_{v \theta n}
\end{array}\right]
$$

\section{Effects of Piezoelectric Control Systems in Turbomachinery Aeroelastic Stability}

Aeroelastic problems can be roughly classified into the categories of response and stability. In stability evaluation or flutter analysis, the input disturbance of the system which is the aerodynamic forces depends on the vibration amplitude of the blade. Theodorsen's unsteady thin-airfoil theory or rational approximation techniques can be used to present the aerodynamic forces as a function of vibration amplitude and its derivatives [7]. Thus, the input disturbance of the system $u_{d}$ depends on $[\xi]$. The generalized aerodynamic force of a 
specified mode can be determined from [8]. The generalized dynamic equation of the beam can be obtained regarding Timoshenko beam theory.

If the compensator input is considered as a linear combination of the modal coordinate and its derivative as

$$
\ddot{q}+2 \varsigma \omega_{n} \dot{q}+\omega_{n}^{2} q=\frac{\left[F_{d}\right]}{M_{s}}+G \omega_{c}^{2} \eta,
$$

the positive position and the strain rate feedback control can be used together. The dependency of $\eta$ on $q$ and $\dot{q}$ leads to the following relation:

$$
\begin{aligned}
& M_{s} \ddot{q}+C_{s} \dot{q}+K_{s} q=F_{a}+G k_{c}^{2} \eta=F_{a}+F(q, \dot{q}) \text {, } \\
& M_{s}=\left[\begin{array}{cc}
1 & -\bar{B} \overline{\mathrm{x}} \\
-\bar{B} \overline{\mathrm{x}} & r_{\theta}^{2}
\end{array}\right], \quad K_{s}=\left[\begin{array}{cc}
2 \zeta_{h s} k_{h} & 0 \\
0 & 2 r_{\theta}^{2} \zeta_{\theta s} k_{h}
\end{array}\right] \text {, } \\
& C_{s}=\left[\begin{array}{cc}
k_{h}^{2} & 0 \\
0 & r_{\theta}^{2} k_{\theta}^{2}
\end{array}\right], \quad K_{c}=\left[\begin{array}{cc}
k_{c}^{2} & 0 \\
0 & r_{\theta}^{2} k_{c}^{2}
\end{array}\right] \text {, } \\
& F_{a}=\frac{1}{\pi \mu}\left\{\begin{array}{c}
\frac{C_{F G}}{b} \\
\frac{C_{M G}}{b^{2}}
\end{array}\right\}, \\
& \left\{\begin{array}{c}
\frac{C_{F G}}{b} \\
\frac{C_{M G}}{b^{2}}
\end{array}\right\}=C(p)\left[\begin{array}{cc}
\int_{0}^{l} \varphi_{i}^{2} \frac{d y}{l} & \bar{B} \\
\bar{B} & \int_{0}^{l} \theta_{i}^{2} \frac{d y}{l}
\end{array}\right]\left\{\begin{array}{c}
\frac{q_{h}}{b} \\
q_{\theta}
\end{array}\right\} \\
& =C(p)\left[\begin{array}{cc}
1-\psi_{\text {norm }} & \bar{B} \\
\bar{B} & 1
\end{array}\right]\left\{\begin{array}{c}
\frac{q_{h}}{b} \\
q_{\theta}
\end{array}\right\}, \\
& C(p)=C_{0} q+C_{1} \dot{q}+C_{2} \ddot{q}+H z
\end{aligned}
$$

in which the auxiliary state variable $z$ is defined so that it satisfies the following equation:

$$
\dot{z}=\dot{q}-\beta z
$$

Writing the above equation in conjunction with a rearranged form of the preceding equation yields the state space equation

$$
\begin{aligned}
\{\dot{\xi}\}= & \left\{\left[\begin{array}{ccc}
0 & {[I]} & 0 \\
-M^{-1} K & -M^{-1} D & -M^{-1} A_{e} H \\
0 & {[I]} & -[\beta]
\end{array}\right]-B_{\text {aero }} G_{\text {aero }}\right\} \\
& \times\left\{\xi_{\text {aero }}\right\}
\end{aligned}
$$

in which

$$
\begin{gathered}
M=M_{s}-A_{e} C_{2}, \quad D=C_{s}-A_{e} C_{1}, \\
K=K_{s}-A_{e} C_{0}, \\
G_{\text {aero }}=\left\{Q G_{c} \quad 0\right\}, \quad\left\{\xi_{\text {aero }}\right\}=\left\{\begin{array}{l}
\xi \\
z
\end{array}\right\} .
\end{gathered}
$$

$A_{e}$ is a factor which depends on mass ratio, bending-torsion coupling [8]. Consider

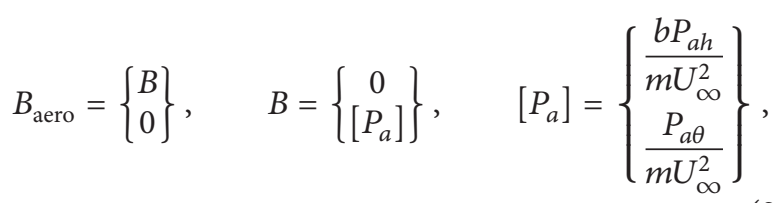

or in the more concise form

$$
[\dot{\xi}]=\left(\left[A_{\text {aero }}\right]-B_{\text {aero }} G_{\text {aero }}\right)\{\xi\}
$$

Obtaining the required parameters of (26) by means of previous relations, the self-excited aeroelastic vibrations of turbomachinery can be reduced by optimized positioning of piezoelectric patches.

\section{Application of Velocity Feedback in Aeroelastic Vibration Control}

This topic is a special case of the optimal control. One can implement vibration control of this condition by velocity feedback or by taking optimal control into account and concerning (8) with velocity feedback design. The main task is $G_{c}$ selection. Some reports exist about the numbers considered for this purpose. Moreover, $\dot{\phi}_{s}(t)$ is the output of the sensor and depends on vibration velocity.

\section{Calculations}

An aeroelastic case was treated in which the structural configuration led to the following characteristics:

$$
\begin{array}{cc}
M_{s}=\left[\begin{array}{cc}
1 & 0.1571 \\
0.1571 & 0.2480
\end{array}\right], & C_{s}=\left[\begin{array}{cc}
0.0025 & 0 \\
0 & 0.0093
\end{array}\right], \\
K_{s}=\left[\begin{array}{cc}
0.0190 & 0 \\
0 & 1.0364
\end{array}\right] \times 10^{6}, & A_{e}=\left[\begin{array}{ll}
0.0016 & 0.0015 \\
0.0015 & 0.0016
\end{array}\right] .
\end{array}
$$

Aerodynamic force coefficients are shown in Table 1. These data were achieved by rational approximation technique after calculation of aerodynamic forces at several reduced frequencies. CFD analysis was performed by FEM approach.

Other input data are $q_{h}(0)=q_{0}$ and $q_{t}(0)=q_{0}$ for initial displacements and $V_{h}(0)=q_{0}$ and $V_{t}(0)=q_{0}$ in the case of velocity excitations. $q_{0}$ can be considered as a value which fits reasonably with plunging or torsional motions in each case.

$$
m_{\text {beam }}=18291.798 \mathrm{~kg} / \mathrm{m}, \quad b=0.11 \mathrm{~m} \text { (beam width) } .
$$


TABLE 1: Aerodynamic force coefficients of the typical aeroelastic case.

\begin{tabular}{lcccc}
\hline & $C_{i}(1,1)$ & $C_{i}(1,2)$ & $C_{i}(2,1)$ & $C_{i}(2,2)$ \\
\hline$C_{0}$ & 32.8508 & 38.0274 & -6.3067 & 11.1821 \\
$C_{1}$ & 1.7196 & 1.0004 & 0.2946 & -0.2616 \\
$C_{2}$ & 2.9262 & 0.1211 & -0.1329 & -0.4225 \\
$C_{3}$ & -3.2556 & -2.0932 & 10.5449 & -0.8498 \\
$C_{4}$ & 26.2369 & 60.9038 & -6.0006 & 16.9342 \\
$C_{5}$ & 0.7657 & -6.9970 & 2.0261 & -5.1781 \\
$C_{6}$ & -6.8888 & 30.7360 & -0.3372 & 8.1604 \\
\hline
\end{tabular}

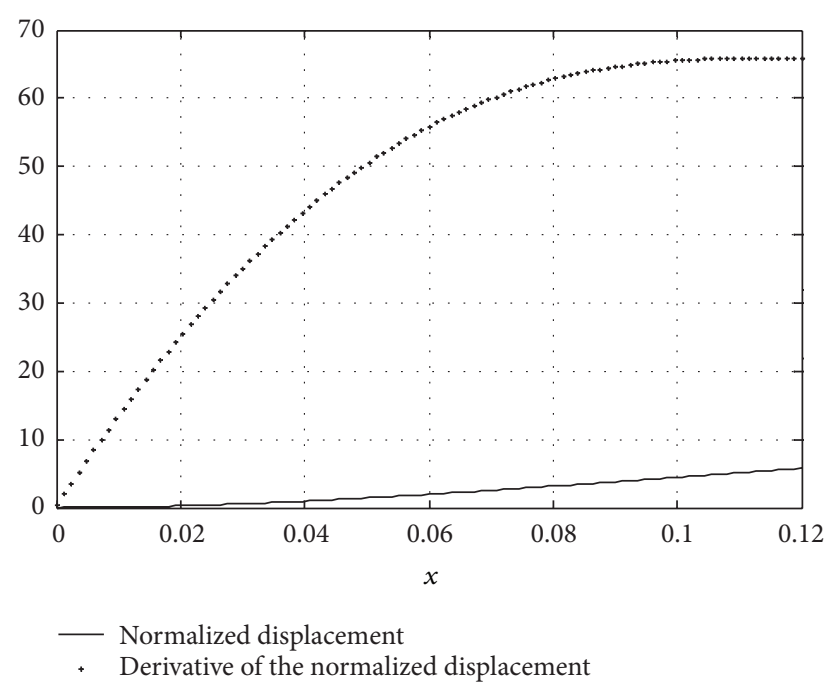

FIGURE 2: Normalized displacement of the beam and its derivative.

Piezoelectric characteristics are $P_{a}=34.2, E_{a}=114 \mathrm{GPa}, q_{0}=$ $[1 \mathrm{~mm}, 1 \mathrm{rad}, 1 \mathrm{~mm} / \mathrm{s}, 1 \mathrm{rad} / \mathrm{s}], d_{31 a}=3 \times 10^{-6}$, and $r_{a}=10^{-3} \mathrm{~m}$ (average distance). So,

$$
\begin{aligned}
& {\left[P_{a}\right]=\left\{\begin{array}{c}
0.001558 \\
0
\end{array}\right\}, \quad B=\left\{\begin{array}{c}
0 \\
0 \\
0.001558 \\
0
\end{array}\right\},} \\
& A(1: 4,1: 4) \\
& =\left[\begin{array}{cccc}
0 & 0 & 1 & 0 \\
0 & 0 & 0 & 1 \\
-1.446 & 50.2304 & -0.0016 & 0.0075 \\
1.1083 & -326.9669 & 0.0116 & -0.0319
\end{array}\right] \text {, } \\
& z=\left\{\begin{array}{llll}
q_{3} & q_{4} & q_{5} & q_{6}
\end{array}\right\} \\
& -M^{-1} A_{e} H=\left\lfloor\begin{array}{lllll}
A_{e} M^{-1} c_{3} & A_{e} M^{-1} c_{4} & A_{e} M^{-1} c_{5} & A_{e} M^{-1} c_{6}
\end{array}\right\rfloor, \\
& {[\beta]=\left[\begin{array}{cccc}
-0.003 & 0 & 0 & 0 \\
0 & -1 & 0 & 0 \\
0 & 0 & -0.02 & 0 \\
0 & 0 & 0 & -0.025
\end{array}\right] \text {. }}
\end{aligned}
$$

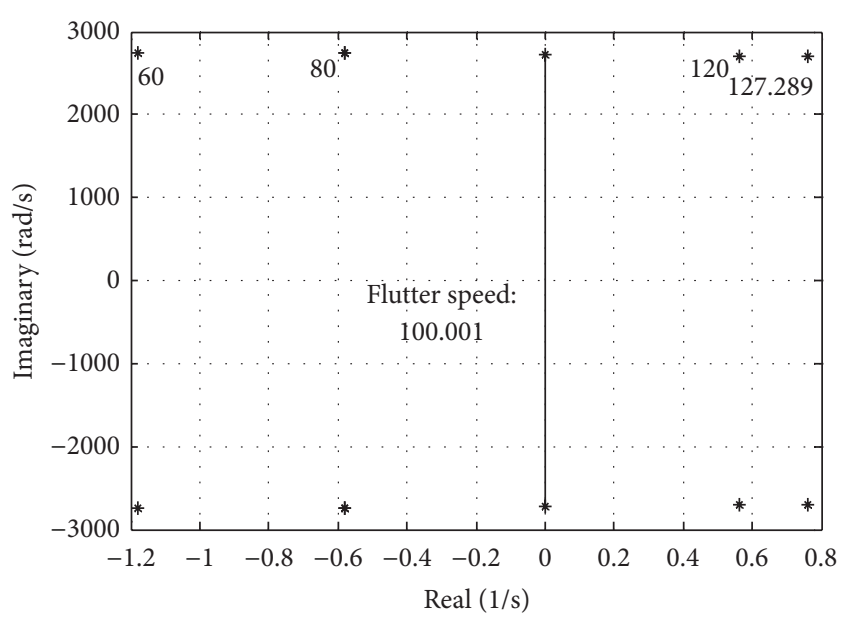

FIGURE 3: Root locus plot without piezoelectric actuator.

\begin{tabular}{|c|c|c|c|c|c|}
\hline & \multicolumn{3}{|c|}{ Amplifier gain } & \multirow{2}{*}{$\begin{array}{c}\text { Flutter } \\
\text { speed } \\
(\mathrm{m} / \mathrm{s})\end{array}$} & \multirow{2}{*}{$\begin{array}{c}\text { Flutter } \\
\text { frequency } \\
(\operatorname{Rad} / \mathrm{s})\end{array}$} \\
\hline & $R$ & $\mu_{x h 1}$ & $\mu_{v h 1}$ & & \\
\hline $\begin{array}{l}\text { Without } \\
\text { piezoelectric }\end{array}$ & 0 & 0 & 0 & 100.0010 & 42990.3611 \\
\hline $\begin{array}{l}\text { With } \\
\text { piezoelectric }\end{array}$ & 0.01 & 1 & 0.3 & 127.2890 & 42991.9285 \\
\hline
\end{tabular}

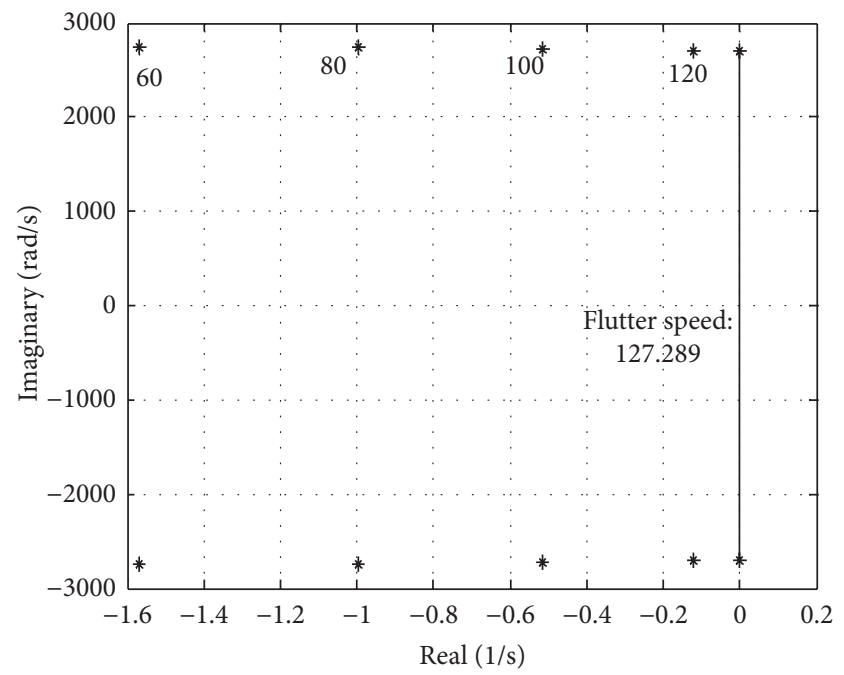

FIgURE 4: Root locus plot with piezoelectric actuator, $R=0.01$, $\mu_{x h 1}=1, \mu_{v h 1}=0.3$.

TABLE 2: The effects of a certain piezoelectric actuator on flutter speed and frequency.

In this typical condition, $[Q]$ was defined based on the importance of plunging displacement. So

$$
Q(i, j)= \begin{cases}1 & i=j=1 \\ 0 & i \neq 1 \text { or } j \neq 1 .\end{cases}
$$

Controllability was checked at the first step for $R=0.01$. The optimum position of the piezoelectric actuator patch or 


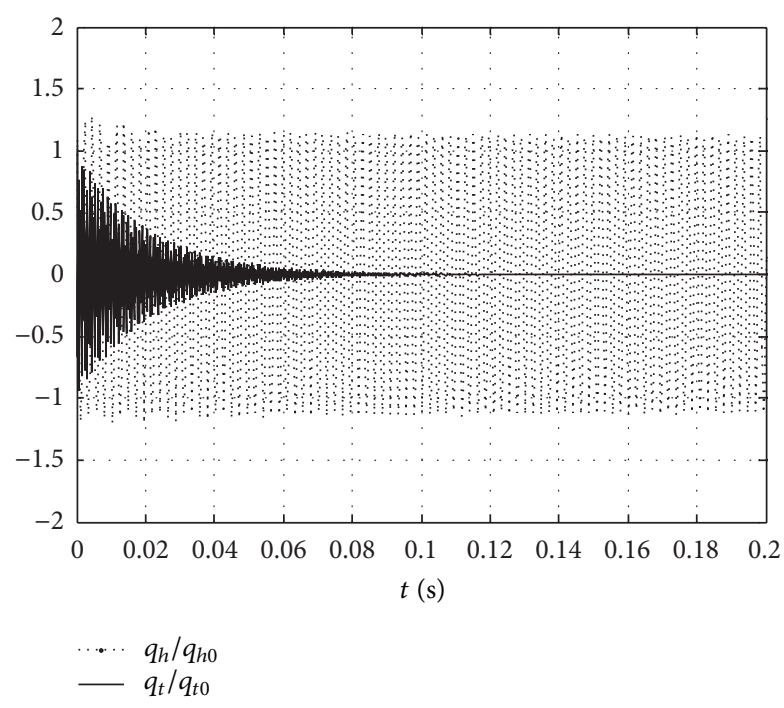

Figure 5: Displacement versus time plot of the generalized coordinate of the beam without piezoelectric attachment, $U=95 \mathrm{~m} / \mathrm{s}$, $q_{h}(0)=q_{h 0}$, and $q_{t}(0)=q_{t 0}$.

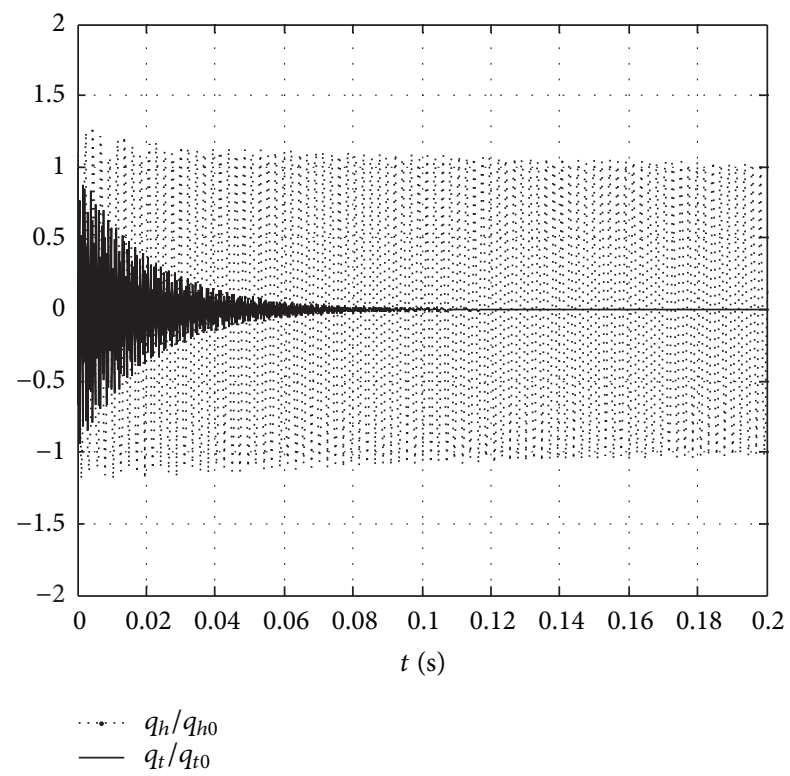

FIGURE 6: Displacement versus time plot of the generalized coordinate of the beam with piezoelectric attachment, $R=0.01, \mu_{x h 1}=1$, $\mu_{v h 1}=0.3, U=95 \mathrm{~m} / \mathrm{s}, q_{h}(0)=q_{h 0}$, and $q_{t}(0)=q_{t 0}$.

layer was obtained by plotting the first normalized mode shape of the overhung beam and its first derivative which is shown in Figure 2. These curves depict that the optimum position is at the root of the blade.

Comparison of Figure 3 with Figure 4 and investigation of Table 2 show that the convenient application of piezoelectric materials moves the real component of the eigenvalues to the right of the root locus plot which is in the more stable conditions which in turn leads to the greater rate of decay. This phenomenon can also be represented graphically by Figures 5, 6, 7, and 8. These figures demonstrate the influence of the various configurations of displacement, velocity

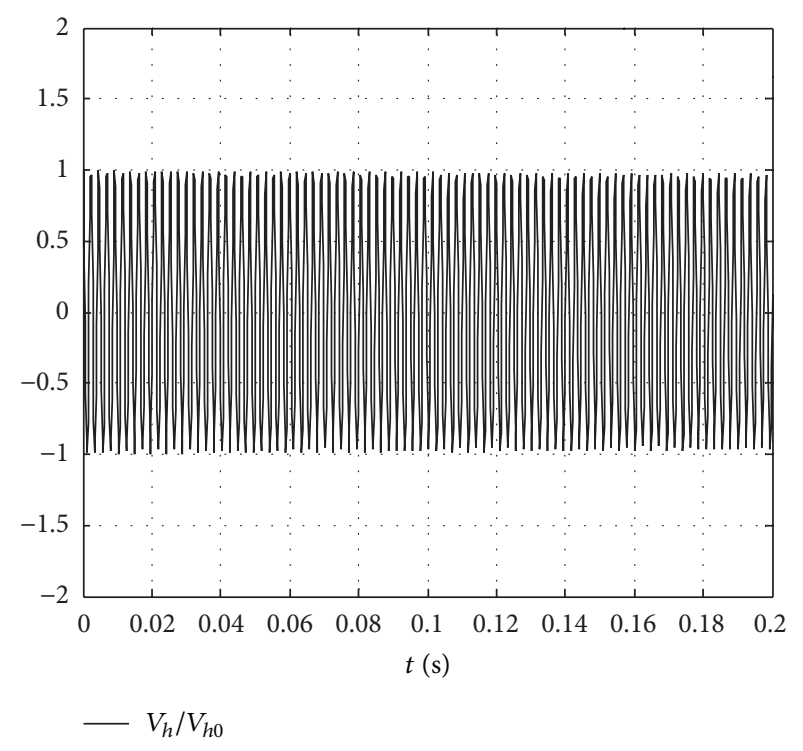

FIGURE 7: Velocity versus time plot of the generalized coordinate of the beam without piezoelectric attachment, $U=95 \mathrm{~m} / \mathrm{s}$ and $V_{q h}(0)=$ $V_{h 0}$.

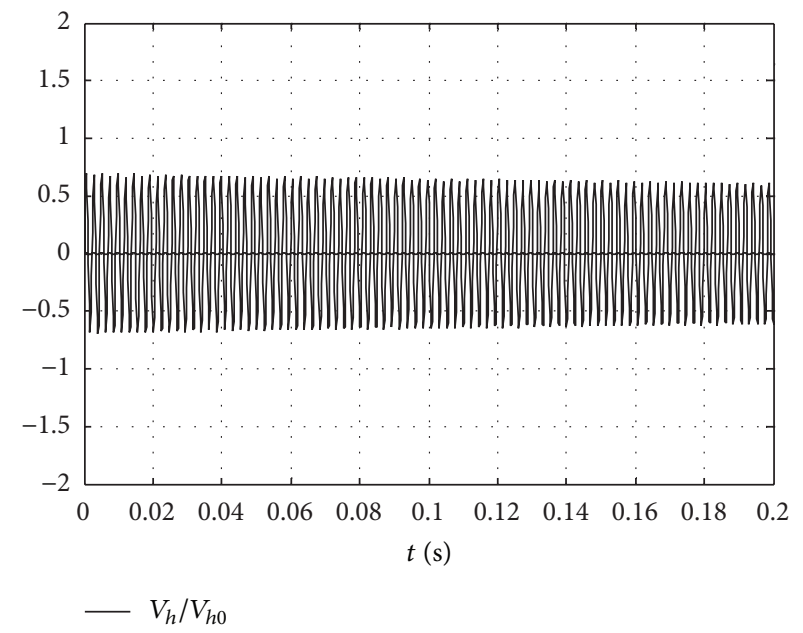

FIGURE 8: Velocity versus time plot of the generalized coordinate of the beam with piezoelectric attachment, $R=0.01, \mu_{x h 1}=1, \mu_{v h 1}=$ $0.3, U=95 \mathrm{~m} / \mathrm{s}$, and $V_{q h}(0)=V_{h 0}$.

gain, and piezoelectric material expenditure which represents graphically the response of the aeroelastic and control system to the unit amplitudes of the desired excitations. The velocity response depicted in Figure 8 is the result of the aeroelastic and control system configured for the aeroelastic model with the output shown in Figure 7. This control system attenuates the velocity amplitude without an appreciable change in displacement. In order to have more displacement decrement, a larger relative value of displacement gain must be considered. The output response of a typical control system of this type is shown in Figure 9. When displacement or velocity gain values are limited, one can manage a control system with greater amount of piezoelectric material by decreasing $R$ value, which results in a significant drop of 


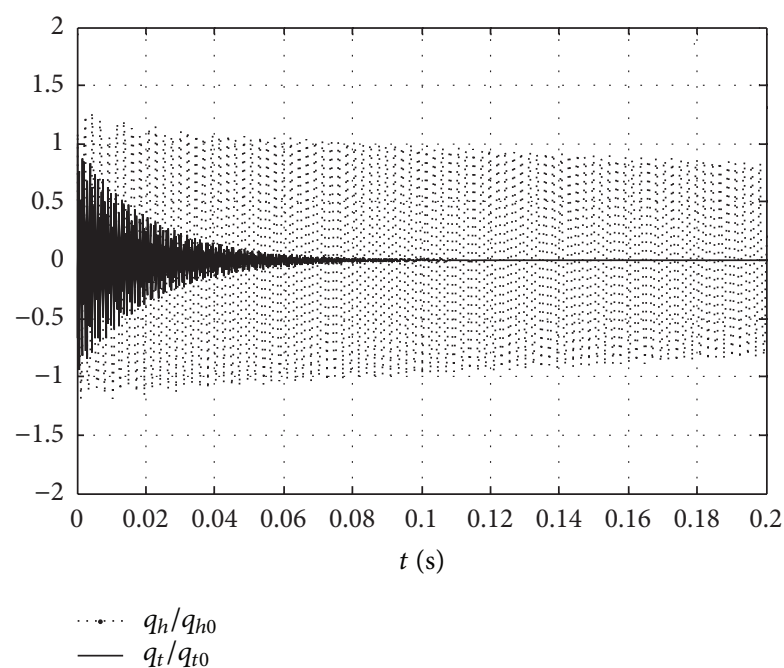

FIGURE 9: Displacement versus time plot of the generalized coordinate of the beam with piezoelectric attachment, $R=0.01, \mu_{x h 1}=30$, $\mu_{v h 1}=0.2, U=95 \mathrm{~m} / \mathrm{s}$, and $V_{q h}(0)=V_{h 0}$.

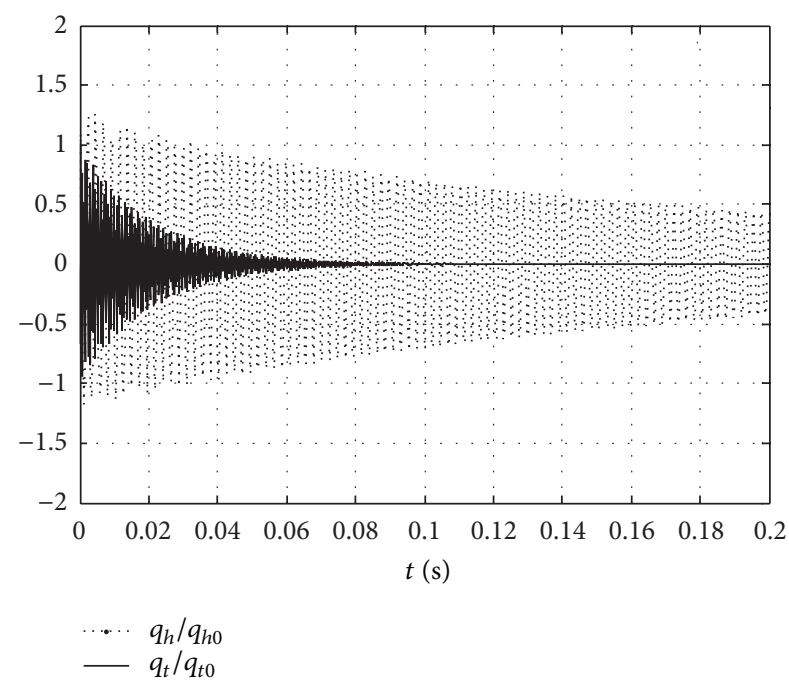

FIGURE 10: Velocity versus time plot of the generalized coordinate of the beam with piezoelectric attachment, $R=0.001, \mu_{x h 1}=30$, $\mu_{v h 1}=0.2, U=95 \mathrm{~m} / \mathrm{s}$, and $V_{q h}(0)=V_{h 0}$.

vibration amplitude with respect to time (Figure 10). This means that vibration amplitude control is preferred against piezoelectric material and energy consumption. The same approach can also be used for pitching vibration attenuation.

\section{Conclusions}

Utilizing the aforementioned capabilities with proper incorporation of piezoelectric elements and optional selection of displacement, velocity attenuation ratio, and control efforts ratio regarding the aeroelastic case can lead to lower aeroelastic self-excited (near flutter) vibration and higher margin of instability. The piezoaeroelastic state space equation is simplified due to the dependency of the aerodynamic forces on the state vector.

\section{Conflict of Interests}

The authors declare that there is no conflict of interests regarding the publication of this paper.

\section{References}

[1] P. Gaudenzi, R. Carbonaro, and E. Benzi, "Control of beam vibrations by means of piezoelectric devices: theory and experiments," Composite Structures, vol. 50, no. 4, pp. 373-379, 2000.

[2] Q. Wang and C. M. Wang, "A controllability index for optimal design of piezoelectric actuators in vibration control of beam structures," Journal of Sound and Vibration, vol. 242, no. 3, pp. 507-518, 2001.

[3] S. Narayanan and V. Balamurugan, "Finite element modelling of piezolaminated smart structures for active vibration control with distributed sensors and actuators," Journal of Sound and Vibration, vol. 262, no. 3, pp. 529-562, 2003.

[4] S. X. Xu and T. S. Koko, "Finite element analysis and design of actively controlled piezoelectric smart structures," Finite Elements in Analysis and Design, vol. 40, no. 3, pp. 241-262, 2004.

[5] J.-C. Lin and M. H. Nien, "Adaptive control of a composite cantilever beam with piezoelectric damping-modal actuators/ sensors," Composite Structures, vol. 70, no. 2, pp. 170-176, 2005.

[6] G. Jacquet-Richardet, Bladed Assemblies Vibration, Laboratoire de Mécanique des Structures, Institut National des Sciences Appliquées, Lyon, France, 1997.

[7] F. M. Karadal, G. Seber, M. Sahin, V. Nalbantoglu, and Y. Yaman, "State space representation of smart structures under unsteady aerodynamic loading," in Proceedings of the 4th International Aerospace Conference, Ankara, Turkey, 2007, AIAC-2007-034.

[8] A. Rahi, M. Shahravi, and D. Ahamdi, "The effects of airfoil camber on flutter suppression regarding Timoshenko beam theory," in Proceedings of the International Conference on Mechanical and Aerospace Engineering, Amsterdam, The Netherlands, 2011.

[9] J. S. Mitchell, An Introduction to Machinery Analysis and Monitoring, chapter 1, Pennwell Publishing, Tulsa, Oklahoma, 2nd edition, 1993. 

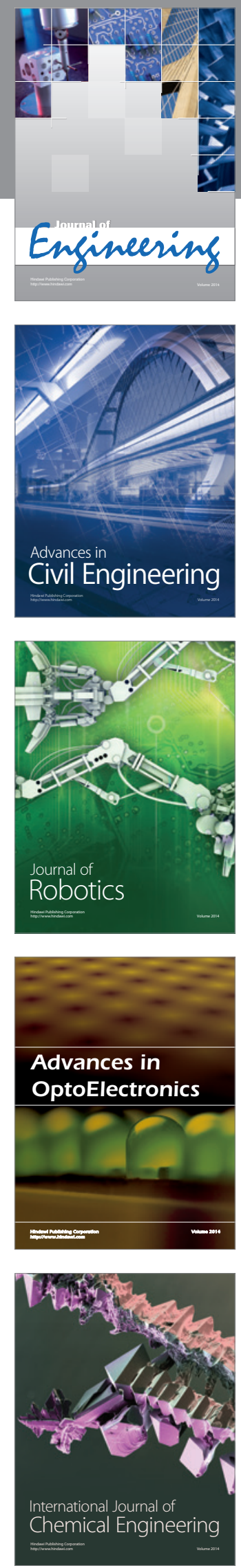

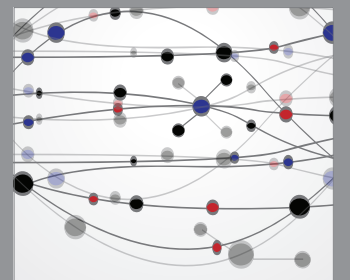

The Scientific World Journal
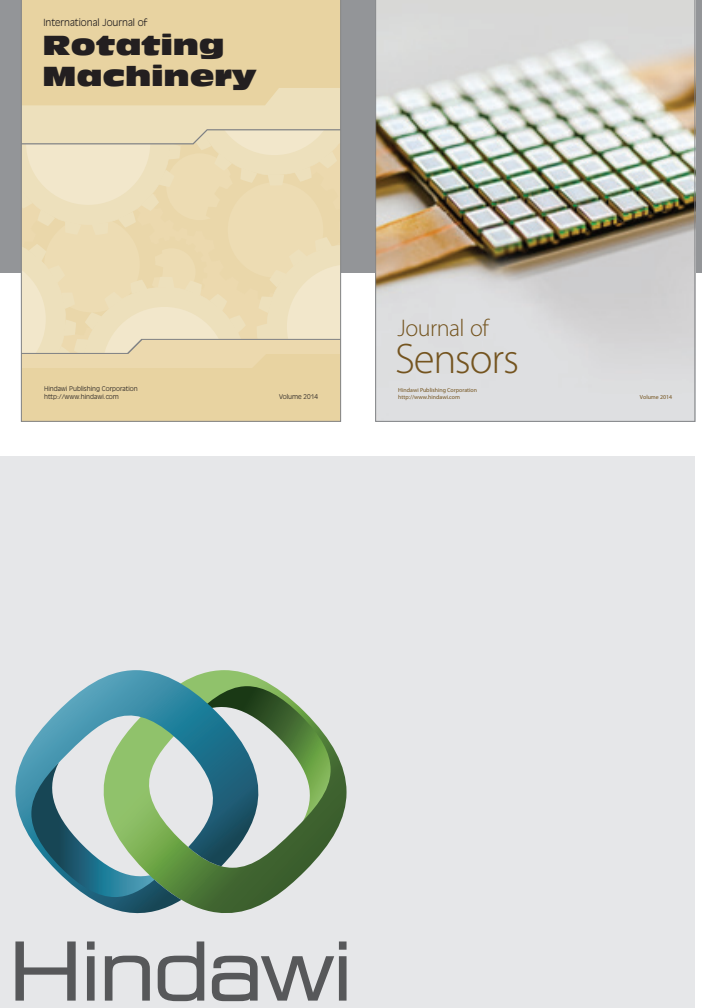

Submit your manuscripts at http://www.hindawi.com
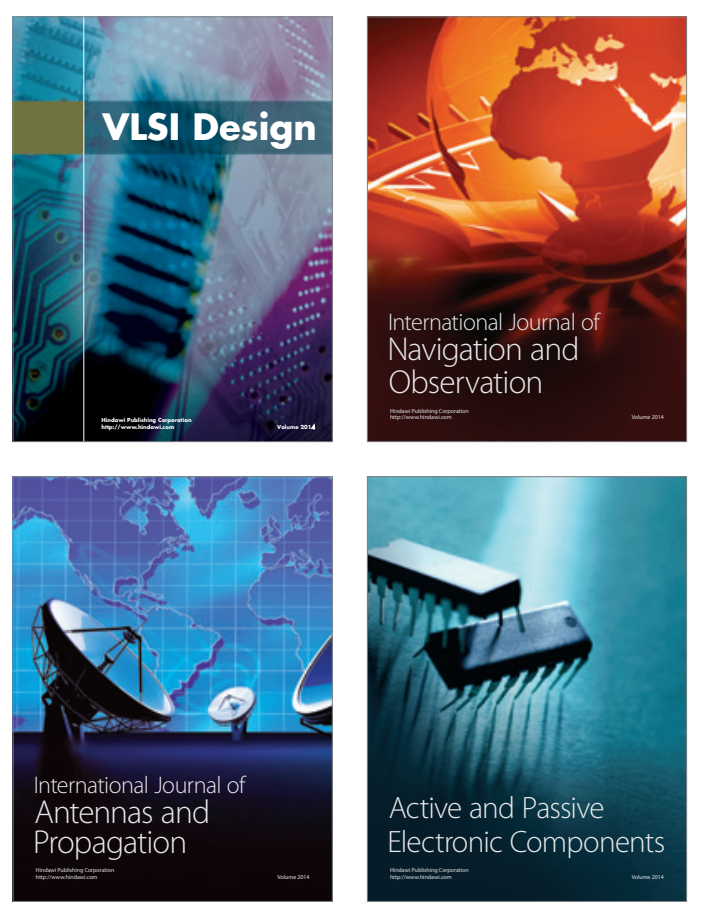
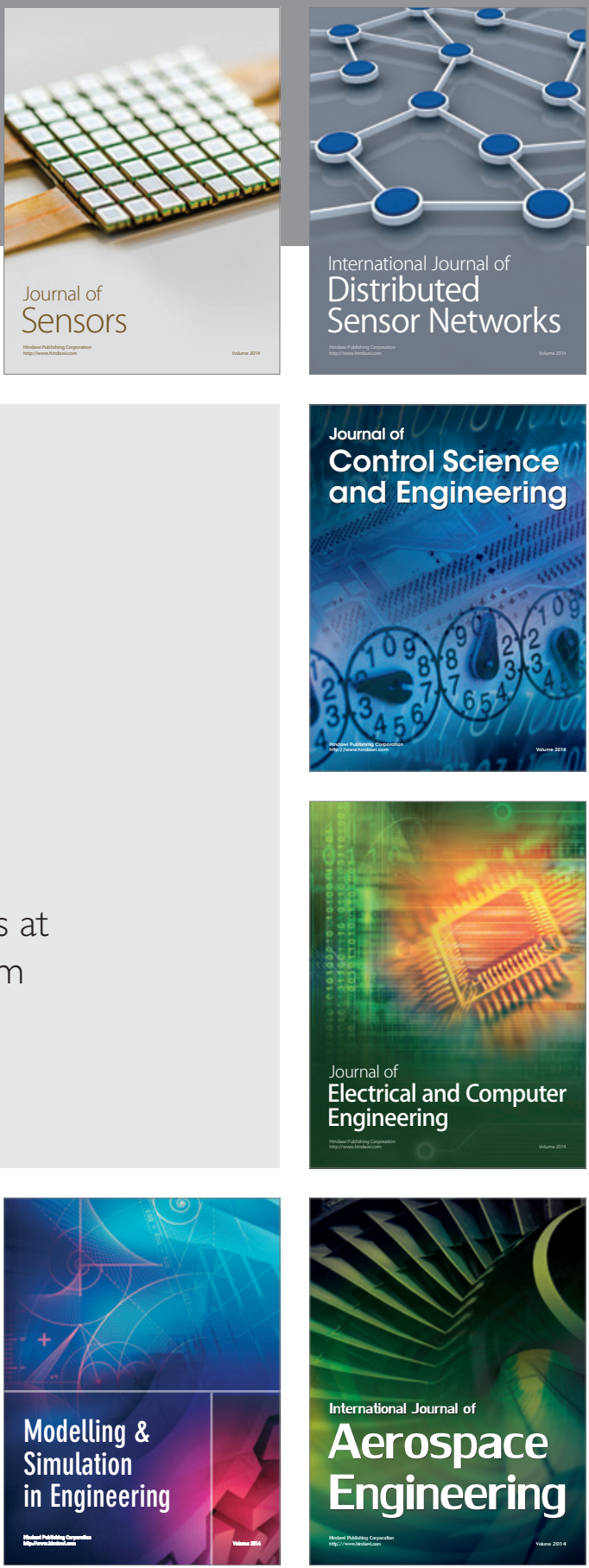

Journal of

Control Science

and Engineering
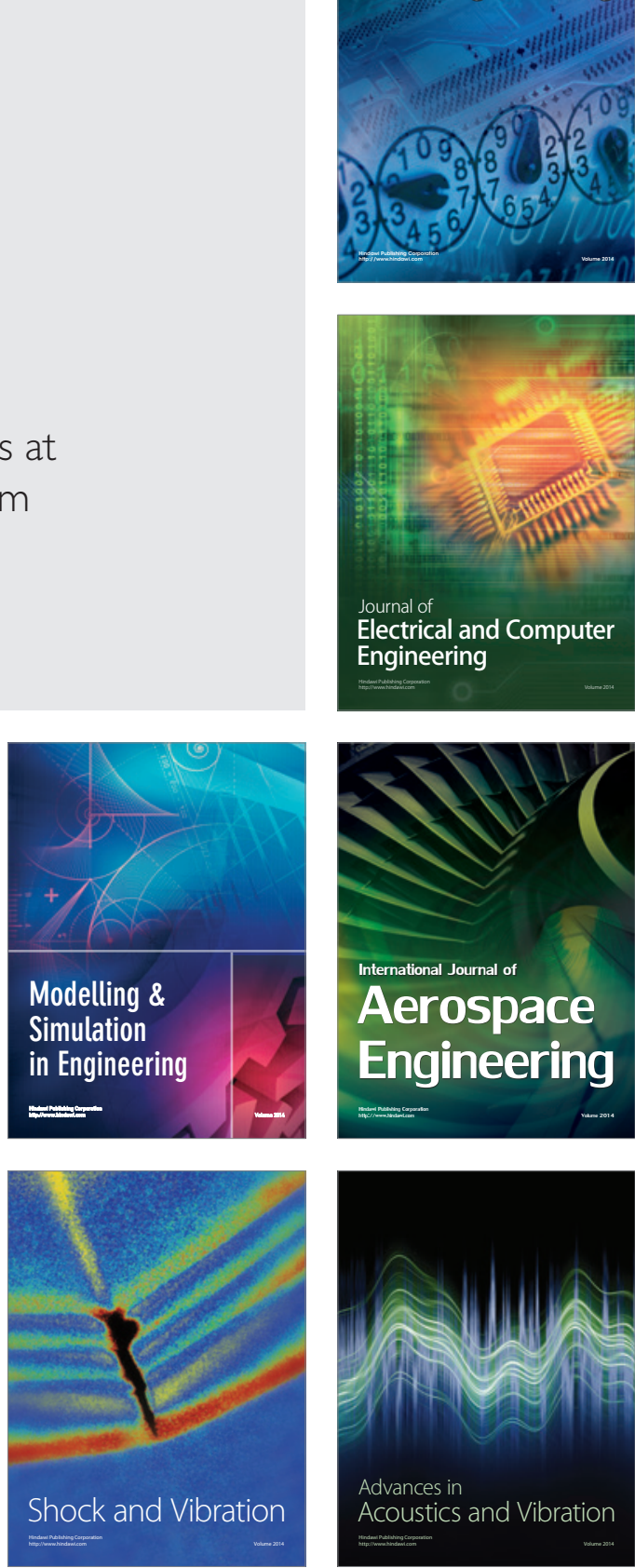